\title{
The Form and Properties of the Tight-Binding Density of States in Two Dimensions. Possible Application to Superconductivity
}

\author{
M. Geadysiewicz, R. Gonczarek and M. Mulak \\ Institute of Physics, Wrocław University of Technology \\ Wybrzeże Wyspiańskiego 27, 50-370 Wrocław, Poland
}

(Received November 5, 2001; revised version March 18, 2002)

\begin{abstract}
The form of the density of states for a tight-binding model with the first and the next-nearest neighbour hoppings for a rectangular planar lattice and a shift of the chemical potential are calculated. This is newly and comprehensively formulated integral form illustrated numerically, helpful in approach to two-dimensional superconducting models. In some particular cases the complete analytical forms of the density of states are obtained. It is shown that for all cases the density of states has a single van Hove singularity of the logarithmic type at the identically defined point. The proper approximate analytical representations are found. The difference between the exact numerical and approximate analytical forms is evaluated and illustrated.
\end{abstract}

PACS numbers: $74.20 .-\mathrm{z}, 74.20 . \mathrm{Fg}$

\section{Introduction}

The concept of combination of the van Hove scenario with the BCS model has already received considerable attention in the context of high temperature superconductivity, e.g. [1-10]. Since this approach seems to be a successful one, the applied formalism is worth investigating more deeply.

This paper is based on the results of our previous work [11] and could be treated as a development and interesting application of the formalism presented therein. 
As it was shown in [11] any anisotropic superconducting system can be considered in the frame of the extended BCS model to which the additional scalar field of the density of states is affixed. This new scalar field, defined for the isotropic momentum space, reflects the distribution of quantum-mechanical states after the conformal space transformation from anisotropic into the isotropic one, including involved mechanisms of internal interactions. The established theory allows us to replace an arbitrary one-particle excitation spectrum with the suitably chosen parabolic form, which, in principle, makes possible to apply the BCS model in explaining high- $T_{\mathrm{c}}$ superconductors (HTSC) properties. Although the precise definition of this scalar field for an arbitrary anisotropic system is a complex problem, it has been proved [11] that for $S$-paired superconducting systems the scalar field reduces itself (within the extended BCS model) to its mean value, which should be identified as the density of states dependent on the quasiparticle energy only. Therefore, in order to investigate those HTSC in which the Cooper pairs are coupled in the pure $S$ state, one can consider the BCS model with the real density of states which fluctuates in the vicinity of the Fermi level and can possess the van Hove singularities.

Recently, in a number of papers, cf. e.g. [11-18], the authors considered a quasi-two-dimensional system of $\mathrm{Cu}$ and $\mathrm{O}$ atoms forming a rectangular planar lattice for which the tight-binding Hamiltonian can be defined. Hence, in the present paper we concentrate on the calculation of the density of states for this system, when the nearest and the second neighbour interactions, with the proper hopping parameters, as well as the occupation parameter, are included into the dispersion relation.

Let us emphasize that a precise calculation of the exact form of the density of states for the discussed dispersion relation is cumbersome. The solutions found so far (see e.g. $[8,12,13,17]$ ) seem to be incomplete or even incorrect [12]. Now, based on the introduced formalism [11], the complete form of the density of states for three particular models and their approximate expressions near the van Hove singularities can be derived analytically. Additionally, their general form can be obtained using numerical methods.

\section{The approximate DOS forms obtained analytically}

Let us consider a two-dimensional system which can be described in terms of the tight-binding one-band Hubbard model, when the one-particle energy spectrum (dispersion relation) is given in the form (cf. [11-13]):

$$
\xi\left(p_{1}, p_{2}\right)=-2 t_{0}\left[\cos \left(p_{1} a_{1}\right)+\cos \left(p_{2} a_{2}\right)\right]+4 t_{1} \cos \left(p_{1} a_{1}\right) \cos \left(p_{2} a_{2}\right)-t_{2},
$$

where $t_{0}$ and $t_{1}$ represent the nearest and next-nearest hopping, respectively. The occupation parameter $t_{2}$ fixes a shift of the Fermi level in the case of the doping and non-half-filled band, and it can be identified with the chemical potential $[5,19]$. 
In order to define the exact density of states form for such system we apply the formalism of the scalar field of the density of states developed recently [11]. According to this formalism the complementary variable $\varphi$ is defined by the relation

$$
\varphi=\arctan (f)
$$

where

$$
f=\frac{\tan \frac{a_{2} p_{2}}{2}}{\tan \frac{a_{1} p_{1}}{2}}\left[\frac{\sin \left(a_{2} p_{2}\right)}{\sin \left(a_{1} p_{1}\right)}\right]^{-\left(2 t_{1} / t_{0}\right)},
$$

and the coordinate system is defined in the new $(\xi, \varphi)$-space. Then, the density of states can be expressed by the formula

$$
\nu(\xi, \eta)=\frac{2}{\pi^{2}} \int_{0}^{\frac{\pi}{2}} J(\xi, \varphi) \mathrm{d} \varphi
$$

where $\eta=2 t_{1} / t_{0}$ and

$$
J(\xi, \varphi)=\left|\begin{array}{cc}
\frac{\partial p_{1}}{\partial \xi} & \frac{\partial p_{1}}{\partial \varphi} \\
\frac{\partial p_{2}}{\partial \xi} & \frac{\partial p_{2}}{\partial \varphi}
\end{array}\right|
$$

is the Jacobian corresponding to the transformation into the new coordinate system. In order to find $J(\xi, \varphi)$ and hence $\nu(\xi, \eta), p_{1}$ and $p_{2}$ as functions of $\xi$ and $\varphi$ have to be derived firstly. From Eqs. (1) and (2), owing to their symmetry relations, $p_{1}$ and $p_{2}$ have the following properties:

$$
p_{1}\left(z, \frac{\pi}{2}-\varphi\right)=p_{2}(z, \varphi) \quad \text { or } \quad p_{1}\left(z, \frac{1}{f}\right)=p_{2}(z, f) .
$$

Below in the paper the parameter $z$ is equal to $\left(\xi+t_{2}\right) / 2 t_{0}$. According to Eq. (6) the integration range in Eq. (4) may be limited to the interval $\left[0, \frac{\pi}{4}\right]$, providing we double the result.

The functions $p_{1}$ and $p_{2}$, however, cannot be derived analytically for an arbitrary $\eta$. In fact, there exist only a few specific cases, e.g. $\eta= \pm 1$ and $\eta=0$, when the exact analytical forms of the density of states are available. As it was shown in [11], formula (4) can be written as

$$
\nu(\xi, \pm 1)=\frac{2}{\pi^{2} t_{0} a_{1} a_{2}} \frac{1}{\sqrt{1 \pm z}} F\left(\frac{\pi}{2}, \sqrt{\frac{3 \mp z}{4}}\right)
$$

and

$$
\nu(\xi, 0)=\frac{2}{\pi^{2} t_{0} a_{1} a_{2}} \frac{1}{\sqrt{8-z^{2}+4 \sqrt{4-z^{2}}}} F\left(\frac{\pi}{2}, \sqrt{\frac{8 \sqrt{4-z^{2}}}{8-z^{2}+4 \sqrt{4-z^{2}}}}\right),
$$

where $F(\phi, \kappa)$ is the first type elliptic integral, which is of the form [20]

$$
F\left(\frac{\pi}{2}, \kappa\right)=\int_{0}^{\frac{\pi}{2}} \frac{\mathrm{d} \alpha}{\sqrt{1-\kappa^{2} \sin ^{2} \alpha}}
$$


The above functions have no poles except singularities of the logarithmic type for $z \rightarrow \pm 1(|z|<1)$ or $z \rightarrow 0$, respectively. To derive the complete form of the density of states for arbitrary values of $\eta$ numerical methods have to be applied. Nevertheless, some approximate results can be achieved analytically when $\eta \ll 1$ by means of the method used to obtain Eq. (8). It should be emphasized, however, that these calculations require a careful analysis, as some results obtained so far are simply incomplete $[8,12]$.

Let us rewrite Eqs. (1) and (2) into the more convenient expressions for the analytical calculations. After introducing the following substitutions:

$$
k_{1}=a_{1} p_{1}, \quad k_{2}=a_{2} p_{2}, \quad x_{1}=\tan \frac{k_{1}}{2}, \quad x_{2}=\tan \frac{k_{2}}{2}, \quad z=\frac{\xi+t_{2}}{2 t_{0}},
$$

they take the forms, respectively

$$
\begin{aligned}
& z=2-\frac{2}{1+x_{1}^{2}}-\frac{2}{1+x_{2}^{2}}+\eta\left[1-\frac{2}{1+x_{1}^{2}}-\frac{2}{1+x_{2}^{2}}+\frac{4}{\left(1+x_{1}^{2}\right)\left(1+x_{2}^{2}\right)}\right] \\
& \tan \varphi=\frac{x_{2}\left(\frac{x_{2}}{1+x_{2}^{2}}\right)^{-\eta}}{x_{1}\left(\frac{x_{1}}{1+x_{1}^{2}}\right)^{-\eta}}
\end{aligned}
$$

It has been already shown [11] that for $\eta=0$ and $\eta= \pm 1$ both $x_{1}$ and $x_{2}$, and consistently $k_{1}$ and $k_{2}$, can be derived analytically from Eqs. (11). Our present aim is to consider $|\eta| \ll 1$ using the perturbation method with respect to the case of $\eta=0$.

$$
\begin{aligned}
& \text { For } \eta=0 \text { we have }[11] \\
& x_{i}^{2}=\frac{z\left(1+f^{2}\right)+\sqrt{\left(1+f^{2}\right) z^{2}+4 f^{2}\left(4-z^{2}\right)}}{2\left(2-z_{i}\right) f^{2-i}},
\end{aligned}
$$

where $i=1,2$ and $f=\tan \varphi$.

For $\eta \neq 0$ and $|\eta| \ll 1 \mathrm{Eq}$. (11) can be transformed to the form

$$
Z=2-\frac{2}{1+x_{1}^{2}}-\frac{2}{1+x_{2}^{2}}, \quad \Phi=\frac{x_{2}}{x_{1}},
$$

where the expressions

$$
\begin{aligned}
Z= & z(1-\eta) \\
& +\eta\left[1-\frac{4 f^{2}(2-z)^{2}}{z\left(1+f^{2}\right)^{2}+4 f^{2}(2-z)+\left(1+f^{2}\right) \sqrt{\left(1-f^{2}\right)^{2} z^{2}+16 f^{2}}}\right], \\
\Phi= & f+\eta f \ln \left\{\frac{1}{f}\left[\frac{2(2-z)\left(f^{2}-1\right)}{z\left(1+f^{2}\right)^{2}+2(2-z)+\sqrt{\left(1-f^{2}\right)^{2} z^{2}+16 f^{2}}}\right]\right\}
\end{aligned}
$$

contain the perturbation contributions calculated with regard to Eq. (12). The structure of reduced Eq. (13) is the same as that for $\eta=0$, but now $Z$ and $\Phi$ are 
functions of $z$ and $\varphi$ dependent on the parameter $\eta$. Nevertheless, we are able to formally solve Eq. (13), employing the results for $\eta=0$ obtained in [11].

Since, for $i=1,2$

$$
\begin{aligned}
& \frac{\partial k_{i}}{\partial \xi}=\left(\frac{\partial k_{i}}{\partial Z} \frac{\partial Z}{\partial z}+\frac{\partial k_{i}}{\partial \Phi} \frac{\partial \Phi}{\partial z}\right) \frac{1}{2 t_{0}} \\
& \frac{\partial k_{i}}{\partial \varphi}=\left(\frac{\partial k_{i}}{\partial \Phi} \frac{\partial \Phi}{\partial f}+\frac{\partial k_{i}}{\partial Z} \frac{\partial Z}{\partial f}\right) \frac{\partial f}{\partial \varphi}
\end{aligned}
$$

hence

$$
\begin{array}{ll}
\frac{\partial Z}{\partial z}=1+\eta f_{1}(z, f), & \frac{\partial Z}{\partial f}=\eta f_{2}(z, f), \\
\frac{\partial \Phi}{\partial f}=1+\eta f_{3}(z, f), & \frac{\partial \Phi}{\partial z}=\eta f_{4}(z, f),
\end{array}
$$

where $f_{j}(z, f)$ denote some functions of $z$ and $f$, whose explicit forms are not essential in the applied perturbation method.

Thus, the Jacobian takes the form

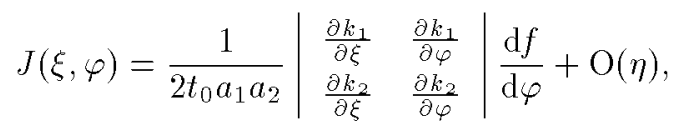

where $O(\eta)$ denotes the remaining terms proportional to $\eta$ and $\eta^{2}$, which, as perturbations, are assumed to be smaller than the main term of the Jacobian and can be neglected. Then, after some transformations and including Eq. (6), the density of states can be written as

$$
\nu(\xi, \eta)=\frac{1}{\pi^{2} t_{0} a_{1} a_{2}} \int_{0}^{1} \frac{1+\Phi^{2}}{\left(1+f^{2}\right) \sqrt{Z^{2}(1-\Phi)^{2}+16 \Phi^{2}}} \mathrm{~d} f
$$

where $Z$ and $\Phi$ are given by Eq. (14). In order to evaluate the approximate behaviour of $\nu(\xi, \eta)$ function at its singular points, we restrict our considerations to the case of $|z| \ll 1$, which is complementary to the case of $|\eta| \ll 1$. After omitting small contributions containing $z$, Eqs. (14) reduce themselves to the form

$$
Z=z+\eta\left(\frac{1-f}{1+f}\right)^{2}, \quad \Phi=f
$$

Now, substituting Eqs. (19) into Eq. (18) we state that the density of states singularities appear only for $z=-\eta$. The proper approximate function can be obtained from Eq. (18), in which the singularities correspond to the lower integration limit when $f \rightarrow 0$.

Hence, we finally obtain

$$
\nu(\xi, \eta)=\frac{1}{\pi^{2} t_{0} a_{1} a_{2}} \ln \frac{8}{|z+\eta|} .
$$


Thus, the logarithmic singularity appears for $\xi=-\left(4 t_{1}+t_{2}\right)$. This conclusion is in agreement with more general results [8].

It is worth emphasizing that the correctness of the above result and hence the perturbation method used, has been confirmed by the independent analytical approximate approach, as well as by exact numerical calculations presented in the next section. It should be also clarified that the linear expansion of Eqs. (11) with respect to $\eta$ leads to the incorrect results, since the logarithmic singularity does not include the influence of additional terms arising for non-zero $\eta$, and its form is the same as for $\eta=0$ (cf. Eq. (20)). The performed approach, however, shows that the density of states possesses a logarithmic singularity at $\xi=-\left(4 t_{1}+t_{2}\right)$ for $\eta \ll 1$, which means that it is very sensitive to even a small correction of the next-nearest hopping.

On the other hand, employing formula (7) and some formal relations [20], we prove that the logarithmic singularities are also present for the cases of $\eta= \pm 1$, when $|z|<1$ and $z \rightarrow \mp 1$, respectively. Then, the density of states reduces itself to the form

$$
\nu(\xi, \pm \eta)=\frac{1}{\pi^{2} t_{0} a_{1} a_{2}} \frac{1}{\sqrt{1 \pm z}} \ln \frac{64}{1 \pm z} .
$$

Therefore, the enhanced logarithmic singularities occur again for $\xi=-\left(4 t_{1}+t_{2}\right)$, if $\eta= \pm 1$, and we may finally conclude that the logarithmic singularities, the same as those for $|\eta| \ll 1$, arise if $\xi=-\left(4 t_{1}+t_{2}\right)$. This is a general statement for an arbitrary $\eta$ as it is proved in the next section.

\section{The general case: analytical approximations and numerical results}

While including into the calculations the values of $\eta$ it has been assumed that they are taken from the interval $-1 \leq \eta \leq 1$ only. This assumption implies a restriction on the parameter $z$, which after taking into account the extreme values of energy $\xi$, Eq. (1), can be written as

$$
-2+\eta \leq z \leq 2+\eta
$$

In order to obtain the general form of the density of states for any value of $\eta$ we employ Eq. (4). Firstly, let us note that the Jacobian can be found for an arbitrary $\eta$ in the following way: differentiating both sides of Eqs. (1) and (3) by $\xi$ and $\varphi$ we get a set of four equations from which the partial derivatives $\partial p_{i} / \partial \xi$ and $\partial p_{i} / \partial \varphi(i=1$ or 2$)$ can be derived. Now, inserting these expressions into Eq. (5), we find the Jacobian in the form

$$
J=\left(2 t_{0} f\right)^{-1}\left[\frac{\sin k_{1}}{\sin k_{2}}\left(1-\eta \cos k_{2}\right)^{2}+\frac{\sin k_{2}}{\sin k_{1}}\left(1-\eta \cos k_{1}\right)^{2}\right]^{-1} \frac{\mathrm{d} f}{\mathrm{~d} \varphi} .
$$

To eliminate $k_{1}$ and $k_{2}$ from the above formula we have to use Eqs. (1) and (3) together with their transformed forms Eqs. (11). Introducing the additional symbols (cf. Eq. (10)) 


$$
v=\frac{x_{2}}{x_{1}}, \quad q=\frac{1}{1+x_{1}^{2}},
$$

we can rewrite the latter of Eqs. (11) as follows:

$$
f=v^{1-\eta} Q^{\eta}
$$

where

$$
Q=v^{2}+q-v^{2} q .
$$

Now, considering the former of Eqs. (11) after the inclusion of the substitutions, Eq. (24), q, being a solution of the appropriate quadratic equation, can be expressed by $v$ and the parameters $z$ and $\eta$ :

$$
\begin{aligned}
q_{1,2} & =1 \\
& -\frac{4(1-\eta)+\left(1-v^{2}\right)(\eta+z) \pm \sqrt{\left(1-v^{2}\right)^{2}(\eta+2)^{2}+16 v^{2}(1+z \eta)}}{4\left[1-\eta-v^{2}(1+\eta)\right]} .
\end{aligned}
$$

Taking into account the definition of $q$ given in Eq. (24) each of the derived forms of $q$ can constitute an allowed solution, providing it satisfies the condition

$$
0 \leq q \leq 1
$$

which is fulfilled, however, only for values of $v$ taken from the interval $[0,1]$ at fixed $\eta$ and $z$. Moreover, the inequalities, Eq. (22), imply the following restrictions:

$$
\begin{aligned}
& (1-|\eta|)^{2} \leq 1+z \eta \leq(1+|\eta|)^{2}, \quad 0 \leq 2 \pm(z-\eta) \leq 4 \\
& -2(1-\eta) \leq z+\eta \leq 2(1+\eta)
\end{aligned}
$$

which, in turn, allow us to estimate $q_{1,2}$ for some specifically chosen values of $v$ :

$$
q_{1,2}=\frac{ \pm|z+\eta|-(z+\eta)}{4(1-\eta)} \text { if } \quad v=0
$$

and

$$
q_{1,2}=\frac{1+\eta \pm \sqrt{1+z \eta}}{2 \eta} \text { if } v=1 .
$$

Hence, we conclude that the only solution which satisfies the inequalities, Eq. (28), for the values of $\eta$ under considerations, is $q_{1}$. Now, employing Eqs. (24)-(27), after the inclusion of Eqs. (10) and (11), we eliminate $k_{1}$ and $k_{2}$ from the Jacobian, Eq. (23). After a few transformations the density of states function can be expressed as follows:

$$
\begin{gathered}
\nu(\xi, \eta)=\frac{2}{\pi^{2} t_{0} a_{1} a_{2}} \\
\times \int_{0}^{1} \frac{Q(v f)^{-1} \mathrm{~d} f}{\left[(1+\eta)\left(1-v^{2}\right)-2 \eta\left(Q-v^{2}\right)\right]^{2}+\left[(1+\eta)\left(1-v^{2}\right) \frac{Q}{v}-2 \eta\left(\frac{Q}{v}-v\right)\right]^{2}},
\end{gathered}
$$


whereas $Q$ reduces itself to the form

$$
\begin{aligned}
Q= & \frac{2 v^{2}}{(1-\eta)-v^{2}(1+\eta)} \\
& \times\left[\frac{2\left(1-v^{2}\right)(1+z \eta)}{\sqrt{\left(1-v^{2}\right)^{2}(z-\eta)^{2}+16 v^{2}(1+z \eta)}+(z-\eta)\left(1-v^{2}\right)}-\eta\right] .
\end{aligned}
$$

In order to find out the numerical form of the density of states function defined by Eq. (31), we have to solve Eq. (25) including Eq. (32), and then derive $v$ as a function of $f$ independently of fixed values of $z$ and $\xi$, when $f$ varies from 0 to 1 . In turn, inserting $f=f(v ; z, \eta)$ into Eqs. (30) and (31), we can compute the integrals and, in consequence, plot the function $\nu(z, \eta)$, Fig. 1.

On the other hand, based on the obtained forms of Eqs. (31) and (32), one can derive analytically an approximate function of the density of states for allowed values of $z$ and $\eta$, when they satisfy the relation $|z+\eta| \ll 1$. Firstly, let us note that if we put $z+\eta=0$, the function $Q$ reduces itself to the expression

$$
Q=v \frac{\sqrt{1+\eta}+v \sqrt{1-\eta}}{\sqrt{1-\eta}+v \sqrt{1+\eta}}
$$

Since the singularities of the density of states in Eq. (31) have their origin in the integration within the small $f(f \ll 1)$ region, we can restrict our considerations by virtue of Eq. (25) to the case of small $v$, which fulfills the additional condition $v \ll \sqrt{1-|\eta|}$

Hence,

$$
\frac{Q}{v}=\sqrt{\frac{1+\eta}{1-\eta}} \text { if } z+\eta=0
$$

and

$$
\frac{Q}{v^{2}}=\frac{2}{1-\eta}\left[\frac{2\left(1-\eta^{2}\right)}{\sqrt{(z+\eta)^{2}+16 v^{2}\left(1-\eta^{2}\right)}+(z+\eta)}-\eta\right] \quad \text { if } \quad z+\eta \neq 0 .
$$

Now, holding consistently the assumption that $z+\eta=0$ in all regular parts of the integrand in Eq. (31), and replacing

$$
f=v\left(\frac{1-\eta}{1+\eta}\right)^{\frac{\eta}{2}}
$$

the density of states function, Eq. (31), for $|z+\eta| \ll 1$ can be approximated as follows (Fig. 2):

$$
\nu(z, \eta)=\frac{1}{\pi^{2} t_{0} a_{1} a_{2}} \frac{1}{\sqrt{1-\eta^{2}}} \ln \frac{8(1-\eta)^{\frac{1+\eta}{2}}(1+\eta)^{\frac{1-\eta}{2}}}{|z+\eta|} .
$$

Finally, one may conclude that the logarithmic singularities in the density of states occur always at $\xi=-\left(4 t_{1}+t_{2}\right)$. Nevertheless, to be in agreement with the 

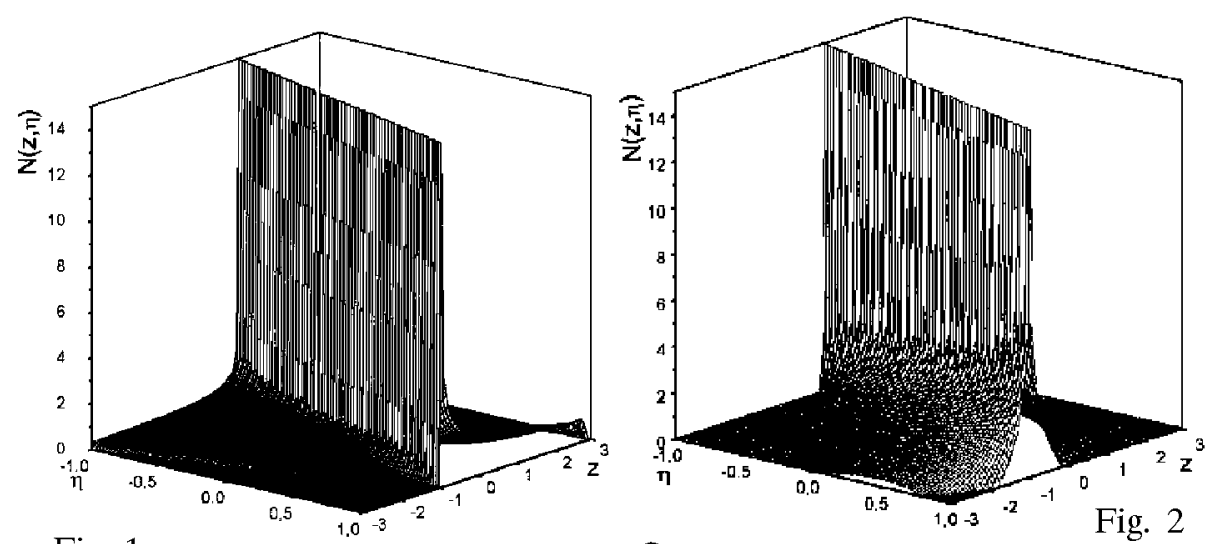

Fig. 1

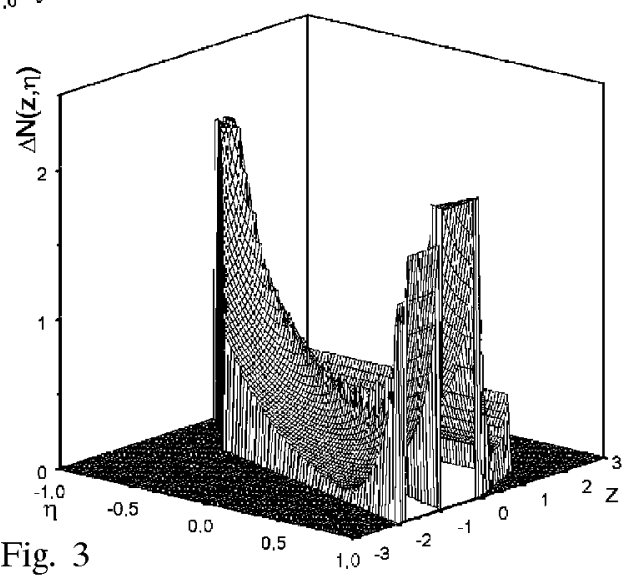

Fig. 2

Fig. 1. The complete form of the density of states in the reduced dimensionless units: $N(z, \eta)=\nu(z, \eta) / \pi^{2} t_{0} a_{1} a_{2}$. The function achieves the infinity for $z+\eta=0$.

Fig. 2. The form of the density of states approximated within the singularity region in the reduced dimensionless units: $N(z, \eta)=\nu(z, \eta) / \pi^{2} t_{0} a_{1} a_{2}$. Negative values of the function are replaced by 0 .

Fig. 3. The difference between the complete and approximated results of the density of states in the reduced dimensionless units within the region of positive values of the approximated formula.

approximation used, the derived formula can be applied only in the close vicinity of singularities, because far from them it becomes negative if $\eta \rightarrow \pm 1$ (Fig. 3).

\section{Conclusions}

The presented results show that the density of states has always one logarithmic singularity for each fixed value of the hopping ratio $\eta=2 t_{1} / t_{0}$, which in the limit $\eta \rightarrow \pm 1$ is intensified by $(1 \mp \eta)^{-\frac{1}{2}}$. However, in the case of larger distances 
from singularities the approximate formula for an arbitrary $\eta$ differs from the exact result substantially, so it can be employed in the limited range. Only the exact formula can be applied successfully in the frame of the extended BCS theory to investigate thermodynamical properties of high- $T_{\mathrm{c}} S$-paired superconductors for all $\eta$ and $z$ under discussion (cf. [11]).

In order to consider systems for which the symmetry of the Cooper pairs wave function is either $p$ - or $d$-like, the scalar field of the density of states (expressed by the Jacobian [11]) has to be included instead of the density of states derived above in the paper.

Note that the scalar field of the density of states encloses a complete information about the distribution function and system symmetry, whereas in the density of states such information becomes averaged over additional coordinates, except $\xi$, i.e., for example, over angular coordinates [11].

\section{References}

[1] J. Labbé, J. Bok, Europhys. Lett. 3, 1225 (1987).

[2] R.S. Markiewicz, Physica C 168, 195 (1990); 183, 303 (1991).

[3] C.C. Tsuei, D.M. Newns, C.C. Chi, P.C. Pattnaik, Phys. Rev. Lett. 65, 2724 (1990); 68, 1091 (1992).

[4] J. Bok, Physica C 209, 107 (1993).

[5] J. Bouvier, J. Bok, in: The Gap Symmetry and Fluctuations in High $T_{\mathrm{c}}$ Superconductors, Eds. J. Bok, G. Deutscher, D. Pavuna, S. Wolf, Plenum Press, New York 1998 , p. 37.

[6] M.J. Getino, M. de Llano, H. Rubio, Phys. Rev. B 48, 597 (1993).

[7] X. Tiefang, B. Guiru, Z. Phys. B 89, 35 (1992).

[8] J.E. Hirsch, D.J. Scalapino, Phys. Rev. Lett. 56, 2732 (1986).

[9] R. Gonczarek, M. Mulak, Phys. Lett. A 251, 262 (1999).

[10] M. Gładysiewicz, R. Gonczarek, M. Mulak, Acta Phys. Pol. A 97, 1039 (2000).

[11] R. Gonczarek, M. Gładysiewicz, M. Mulak, Int. J. Mod. Phys. B 15, 491 (2001).

[12] R. Baquero, D. Quesada, C. Trallero-Giner, Physica C 271, 122 (1996).

[13] R.S. Markiewicz, J. Phys. Chem. Solids 58, 1179 (1997).

[14] J.E.F. Assad, M. Imada, D.J. Scalapino, Phys. Rev. Lett. 77, 4592 (1996).

[15] J. Yu, A.J. Freeman, J.H. Xn, Phys. Rev. Lett. 58, 1035 (1997).

[16] L.F. Mattheiss, Phys. Rev. Lett. 58, 1028 (1997).

[17] C.P. Enz, Phys. Rev. B 54, 3589 (1996).

[18] D.J. Scalapino, Phys. Rep. 250, 329 (1995).

[19] H.Q. Lin, J.E. Hirsch, Phys. Rev. B 35, 3359 (1987).

[20] I.S. Gradstein, I.M. Ryzhik, Tables of Integrals, Sums, Series and Products, Izd. Nauka, Moscow 1962 (in Russian). 